\title{
On E-pseudovarieties of finite regular semigroups
}

\author{
J. D. RODGERS
}

(Received 9 January 2007)

Birkhoff [3] introduced the concept of a variety of algebras, a class of algebras all of the same type, closed under the taking of homomorphic images, subalgebras of the same type and direct products. Birkhoff showed that such classes were defined by sets of identities (for example, within the class of all semigroups, the collection of all commutative semigroups is a variety defined by the identity $x y=y x$ ). Conversely, any class of algebras, all of the same type, all of which satisfy the same set of identities, is a variety. Thus began the study of universal algebra. Over the years, numerous classes of algebras were studied using the new tools of universal algebra: groups, rings, lattices and, importantly for us, semigroups.

The survey by Evans [6] described the research into varieties of semigroups up to the early 1970s. In the mid-1970s a strong relationship was established between certain classes of finite semigroups and the theory of formal languages and automata, which had been developed in the previous two decades. Since these classes consisted of finite semigroups, now only finite direct products were permitted. It is relatively easy to show that such classes of finite semigroups need not form varieties of semigroups, and a new theory of pseudovarieties was developed, initially by Eilenberg and Schützenberger [5]. Their first paper 'On pseudovarieties' was part of a larger study on languages and automata by Eilenberg [4].

The original work of Eilenberg and Schützenberger was based upon the discovery that pseudovarieties may be defined by sequences of identities (as opposed to sets of identities in the case of varieties). In the early 1980s, Reiterman [11] showed, using a topological argument, that pseudovarieties may be defined by sets of pseudoidentities. Shortly thereafter, Ash [2] proved that pseudovarieties consist of the finite members of certain classes of algebras called 'generalized varieties'.

In the late 1980s and early 1990s, existence varieties (or e-varieties) of regular semigroups were defined (independently) by Hall [7] and by Kadourek and

Thesis submitted to RMIT University, March 2007. Degree approved, July 2007.

Supervisor: Dr Graham T. Clarke.

(c) 2008 Australian Mathematical Society 0004-9727/08 \$A2.00+0.00 
Szendrei [8]. E-varieties allow one to study classes of regular semigroups using universal algebraic techniques.

It seems natural to marry the two concepts of e-variety and pseudovariety in a new notion of e-pseudovariety. Mangold introduced e-pseudovarieties of finite regular semigroups in her doctoral thesis [9]. A class of finite regular semigroups forms an e-pseudovariety if it is closed under the taking of homomorphic images, regular subsemigroups and finite direct products.

In this thesis we further develop the theory of e-pseudovarieties, with particular emphasis on congruences on certain sublattices of the lattice of e-pseudovarieties of finite regular semigroups.

An overview of the thesis follows.

In Chapter 1, we discuss those concepts of semigroup theory, lattice theory and universal algebra that are used throughout the remainder of the thesis. In this chapter, we also survey those aspects of the theory of pseudovarieties and e-varieties that are important to this work. Finally, generalized varieties are discussed.

In Chapter 2, we introduce generalized e-varieties. We establish the important result that all e-pseudovarieties of $E$-solid or locally inverse semigroups consist of the finite members of some generalized e-variety (Theorem 2.3.7). We are later able to refine this result in an interesting direction (Corollary 2.4.15).

The final results of Chapter 2 involve properties of certain sublattices of the lattice of e-pseudovarieties of finite regular semigroups. We demonstrate (in a result proved originally by Agliano and Nation for pseudovarieties [1]) that many important lattices of e-varieties are isomorphic to certain related lattices of e-pseudovarieties (Theorem 2.4.17). We then describe a complete lattice homomorphism between certain lattices of generalized e-varieties and lattices of e-pseudovarieties (Theorem 2.4.18).

Chapter 3 is the final chapter in this thesis. This chapter is heavily influenced by research carried out by Pastijn and Trotter [10]. We demonstrate that similar results exist for e-pseudovarieties to those that were obtained by Pastijn and Trotter for pseudovarieties.

Some results from Chapters 2 and 3 will appear in a paper in the Proceedings of the Sydney University Semigroups Conference of June 2005 [12]. Two other papers are currently in preparation, one based on additional results in Chapter 2 [13] and the other on additional results in Chapter 3 [14].

\section{References}

[1] P. Agliano and J. B. Nation, 'Lattices of pseudovarieties', J. Aust. Math. Soc. (Series A) 46 (1989), $177-183$.

[2] C. J. Ash, 'Pseudovarieties, generalized varieties and similarly described classes', J. Algebra 92 (1985), 104-115.

[3] G. Birkhoff, 'On the structure of abstract algebras', Proc. Camb. Phil. Soc. 31 (1935), 433-454.

[4] S. Eilenberg, Automata, languages and machines, Vol. B (Academic Press, New York, 1976).

[5] S. Eilenberg and M. P. Schützenberger, 'On pseudovarieties', Adv. Math. 19 (1976), 413-418.

[6] T. Evans, 'The lattice of semigroup varieties', Semigroup Forum 2 (1971), 1-43. 
[7] T. E. Hall, 'Identities for existence varieties of regular semigroups', Bull. Austral. Math. Soc. 40 (1989), 59-77.

[8] J. Kadourek and M. B. Szendrei, 'A new approach in the theory of orthodox semigroups', Semigroup Forum 40 (1990), 257-296.

[9] M. Mangold, 'E-varieties and E-pseudovarieties of regular semigroups', Doctoral Thesis, Monash University, 1995.

[10] F. Pastijn and P. G. Trotter, 'Complete congruences on lattices of varieties and of pseudovarieties', Internat. J. Algebra Comput. 8 (1998), 171-201.

[11] J. Reiterman, 'The Birkhoff theorem for finite algebras', Algebra Universalis 14 (1982), 1-10.

[12] J. D. Rodgers, 'Some applications of generalised existence varieties', Proceedings of the June 2005 Sydney University Semigroups Conference to appear.

[13] _ 'Generalised e-varieties', in preparation.

[14] _ 'The lattice of e-pseudovarieties of finite regular semigroups', in preparation.

J. D. RODGERS, School of Mathematical and Geospatial Sciences, RMIT University, 124 Latrobe St, Melbourne, VIC 3000, Australia e-mail: rodgers.jd@gmail.com 